\title{
Eine dicke Haut kann nicht schaden
}

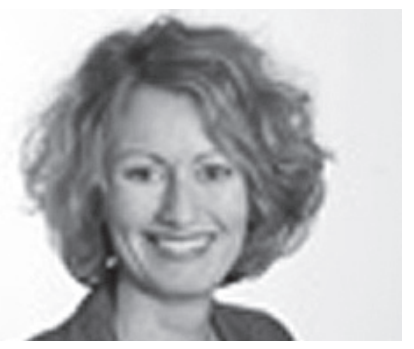

VON SABINE PROHASKA Sabine Prohaska ist Inhaberin des Trainings- und Beratungsunternehmens "seminar consult prohaska« in Wien.

Internet

www.seminarconsult.at

\begin{abstract}
Für viele Organisationen und Betriebe gilt: Das Arbeitspensum und den Arbeitsdruck können die Mitarbeitenden nicht senken - und oft auch ihre Vorgesetzten nicht. Was jedoch erhöht werden kann, ist die "Resilienz", also die Widerstandsfähigkeit gegen Stress aller Art. Mitarbeitenden zu einer "dickeren Haut" zu verhelfen - mit dieser Aufgabe sehen sich zunehmend die Führungskräfte in der Sozialwirtschaft konfrontiert.
\end{abstract}

Im Alltagstrubel ist uns oft nicht bewusst, wie stark die Neuen Medien in sehr kurzer Zeit - nicht einmal zwei Jahrzehnten - neben unserem Arbeitsleben auch unser Verhalten verändert haben. »Ich muss stets und überall erreichbar sein. Und wenn nicht? Dann ihre Strategien überdenken und ihre Art, Aufgaben zu lösen. Für ihre Mitarbeiterinnen und Mitarbeiter bedeutet dies: An sie werden immer häufiger neue Anforderungen gestellt. Und sie müssen permanent gewohnte Denk- und Verhaltensmuster aufgeben.

\section{"Mobile Kommunikation kann zu ständiger ,Rufbereitschaft ‘ führen"}

muss ich wenigstens so schnell wie möglich reagieren." Das hat sich nicht nur zu einem Lebensgefühl vieler Menschen entwickelt. In vielen Jobs sehen sich Berufstätige tatsächlich mit dieser Erwartung konfrontiert; sei es seitens ihrer Kollegen, Chefs oder Kunden, wodurch zunehmend auch die Grenzen zwischen Beruf und Freizeit verschwimmen.

Doch nicht nur wegen der permanenten »Rufbereitschaft « fällt es immer mehr Menschen schwer, einmal ganz abzuschalten. Hinzu kommt: Sie stehen tatsächlich in immer kürzeren Zeitabständen vor neuen Herausforderungen und das nicht nur am Arbeitsplatz. Denn bekannt ist: Die Unternehmen müssen heute in immer kürzeren Zeitabständen

\section{Mitarbeitende sehen oft den Sinn nicht}

Viele Mitarbeitende und Führungskräfte sehen sich zunehmend mit unrealistischen Zielvorgaben konfrontiert. Die Folge: Viele Berufstätige versuchen einfach nur noch zu funktionieren - ohne sich mit den Zielvorgaben zu identifizieren. Denn diese sind meist nicht nur hoch: Sie sind auch sehr abstrakt. Wie soll sich ein Mitarbeiter zum Beispiel mit der Vorgabe »Die Nutzerzahl muss um fünf Prozent steigen « identifizieren, wenn er nicht weiß, wozu dies nötig ist - außer für ein besseres Ergebnis auf der Einnahmenseite? Sinnstiftende Zusammenhänge gehen so verloren. 


\section{Was man tun kann}

Das Burn-out-Syndrom stellt (noch) keine anerkannte medizinische Diagnose dar. Vor diesem Hintergrund fand im Januar 2011 mit führenden österreichischen Expertinnen und Experten auf dem Gebiet der Psychiatrie, Neurologie, Inneren Medizin, Psychologie und Allgemeinmedizin ein interdisziplinäres Treffen statt.

Die Zielsetzung der Veranstaltung war, aktuelle Aspekte zur Diagnose und Therapie des Burn-out-Syndroms zu diskutieren und Empfehlungen für die Praxis zu erarbeiten. Eine zwölfseitige Zusammenfassung der Ergebnisse dieser Diskussion dient gleichzeitig als übersichtlicher Leitfaden, der behandelnden Personen als Informationsgrundlage und Orientierungshilfe für die Prävention, Diagnosestellung und Behandlung eines Burn-out-Syndroms dienen soll. In dieser Publikation werden einige Empfehlungen zur Prävention und Behandlung des Burn-out-Syndroms genannt:

- Ohne chronische, vorwiegend berufsspezifische Belastungssituation entsteht kein Burn-out-Syndrom, wenngleich außerberufliche Belastungen infolge der Verknüpfung zwischen Privat- und Berufsleben eine große Rolle spielen.

- Das Burn-out-Syndrom zeigt in der Regel einen phasenhaften Verlauf und verfügt über eine hohe Komorbidität mit Depressionen, Sucht- und psychosomatischen sowie internistischen

\section{Die Widerstandsfähigkeit steigern}

Alle diese Punkte - permanente Rufbereitschaft, erhöhter Leistungsdruck, Sinnverlust - verursachen bei vielen Mitarbeitenden ein Gefühl der Überforderung, das im schlimmsten Fall zu einem Burn-out führt.

Doch warum fällt bei gleichen Arbeitsbedingungen Mitarbeiter Müller wegen eines »Burn-outs « für ein halbes Jahr aus, während die Kollegin Maier nur ab und zu klagt »Manchmal ist es ganz schön stressig " und sich dann wieder an die Arbeit macht? Mit dieser Frage beschäftigt sich die Resilienzforschung. Der Begriff »Resilienz« leitet sich vom englischen Wort »resilience« $\mathrm{ab}$ und bedeutet so viel wie »Widerstandsfähigkeit«.

Die Resilienzforschung befasst sich mit Fragen wie: Was hält uns gesund? Und: Was gibt uns Kraft? Oder anders formuliert: Warum haben manche Men-
Erkrankungen, die im Zuge der Diagnosestellung in die therapeutischen Überlegungen einbezogen werden müssen.

- Prävention und Therapie umfassen multimodal-interdisziplinäre Zugänge, die sich primär an funktionelle somatische Syndromen und Stressbewältigungsfertigkeiten richten.

- Eine Rezidivprophylaxe des Burn-outSyndroms sollte Warnzeichen wie beginnende Erschöpfung oder übertriebenen Energieeinsatz rechtzeitig identifizieren. Die Früherkennung von Vorstadien auf persönlicher Ebene, in der Arbeitsumgebung -- meist durch den Allgemeinmediziner, durch Arbeitsmedizin oder Arbeitspsychologie - muss als stetiger Prozess verstanden werden.

- Als nichtpharmakologische Strategien empfehlen sich psychotherapeutische Maßnahmen einschließlich kognitivverhaltenstherapeutische Techniken, Verbessern der sozialen Kompetenzen und der Emotionsregulation, Stressmanagement-Training ergänzt durch psychodynamische Ansätze.

Die Publikation kann kostenlos aus dem Internet heruntergeladen werden: http:// www.seminarconsult.at/d/2011_DV_Experten_Statement_Burnout_\&_Depression.pd $\bar{f}$

schen so etwas wie eine »dicke Haut", an der Belastungen scheinbar spurlos abprallen, und andere nicht? Und was mindestens ebenso interessant ist: Woher kommt oder wie entsteht diese »Schutzschicht«?

\section{Sieben Schlüsselqualifikationen}

Wissenschaftliche Studien belegen, dass Menschen, die sich durch eine hohe Widerstandsfähigkeit auszeichnen, meist über folgende sieben Eigenschaften oder Persönlichkeitsmerkmale verfügen:

1. Problemlösefähigkeit: Widerstands- fähige Menschen denken lösungsorientiert; sie beschäftigen sich planend, aber nicht besorgt mit ihrer Zukunft.

2 (Selbst-)Verantwortung überneh- men: Widerstandsfähige Menschen lassen sich nicht in eine Opferrolle drängen.
3. Selbstwirksamkeit: Widerstands- fähige Menschen akzeptieren die Dinge, so wie sie sind, aber lassen diese nicht so.

1 Selbstwertgefühl: Widerstands- fähige Menschen glauben an sich und an das, was sie tun.

5. Soziale Kompetenzen: Wider3. standsfähige Menschen schotten sich bei Stress nicht ab.

6. Stressbewältigungsstrategien: Widerstandsfähige Menschen haben die Fähigkeit, sich Unterstützung $\mathrm{zu}$ organisieren - und sie können sich entspannen.

7. Positives Denken: Widerstandsfä- hige Menschen reagieren auf neue Herausforderungen nicht panisch, sondern denken sich: Irgendwie schaffe ich das schon.

\section{Entwicklungshelfer für die Persönlichkeit}

Doch wie können Menschen die vorgenannten Fähigkeiten und Eigenschaften entwickeln? Die positive Nachricht: Sie schlummern bereits in fast jedem von uns. Die negative Nachricht: Allein ohne externe Unterstützung - fällt es den meisten Menschen schwer, diese zu aktivieren. Denn dies setzt die Erkenntnis voraus: Wie reagiere ich regelmäßig in gewissen Situationen? Zum Beispiel bei neuen Aufgaben oder Herausforderungen? Oder wenn wichtige Entscheidungen zu treffen sind? Des Weiteren: Warum reagiere ich so und nicht anders?

Selbstverständlich können wir uns diese Fragen auch alleine stellen. Doch oft finden wir dann nicht die richtigen Antworten. Zum Beispiel, weil wir gar nicht registrieren, dass wir in vergleichbaren Situationen stets ähnlich reagieren. Oder weil uns unser Verhalten als so normal erscheint, dass wir uns gar nicht vorstellen können, anders zu reagieren.

Deshalb empfiehlt es sich meist, wenn man festgefahrene Verhaltensmuster durchbrechen möchte, sich einen professionellen Sparringpartner zu suchen, zum Beispiel einen Coach, der einen auf die eigenen blinden Flecken hinweist. Denn nur dann lassen sich auch - alleine oder mit Unterstützung des Coachs - 


\section{Was versteht man eigentlich unter Resilienz?}

Resilienz ist die Fähigkeit von Individuen, erfolgreich mit belastenden Lebensereignissen und Krisen umzugehen. Resilienz wird auch als "seelische Widerstandskraft" verstanden. Der Begriff wurde geprägt aus den Erkenntnissen von Langzeitstudien (v. a. E. Werner), bei denen sich zeigte, dass einem Teil von Kindern und Jugendlichen trotz schwierigster Lebensumstände eine körperlich und seelisch gesunde Entwicklung gelang. Resilienz ist keine angeborene Persönlichkeitseigenschaft, sondern wird im Verlauf des Lebens entwickelt; von besonderer Bedeutung sind dabei die frühen Lebensjahre. Das Kind wird als aktiver "Bewältiger" und (Mit-) Gestalter seines Lebens gesehen; die Fähigkeit der seelischen Widerstandskraft entwickelt sich aus der Interaktion mit den Bezugspersonen und realen positiven Bewältigungserfahrungen. Resilienz ist eine dynamische Fähigkeit, die sich im Lebenslauf verstärken oder abbauen und in verschiedenen Lebensbereichen unterschiedlich stark ausgeprägt sein kann. Empirisch konnte eine Reihe von protektiven (Resilienz-) Faktoren identifiziert werden, die die Widerstandskraft gegenüber Belastungen stärken. Den wichtigsten Schutz stellt eine stabile emotionale Beziehung zu mindestens einer primären Bezugsperson dar; kompensatorisch können diese Bedeutung auch spätere (professionelle) Bezugspersonen wie Erzieher/innen oder Lehrer/innen übernehmen. Weitere resilienzfördernde Faktoren sind: soziale Unterstützung außerhalb der Familie, ausgeprägte Selbststeuerungsbzw. Selbstregulationsfähigkeiten, soziale Kompetenzen, Problemlösefähigkeiten und ein positives Selbstwirksamkeitserleben. Das Resilienz-Konzept steht in enger Verbindung zum Paradigmenwechsel in den Sozialwissenschaften hin zu einer Ressourcenorientierung und zu Ansätzen der Salutogenese (Antonovsky). Die Fähig keit der Resilienz kann in den Sozialisationsinstanzen Kindertageseinrichtung und Schule gezielt gefördert werden, hierzu liegen einige wenige Programme vor. Von besonderer Bedeutung ist dabei ein multimodales Vorgehen, das die Oualifizierung pädagogischer Fachkräfte, die Arbeit mit Kindern und Eltern sowie eine institutionelle Vernetzung umfasst.

Klaus Fröhlich-Gildhoff/

Maike Rönnau-Böse

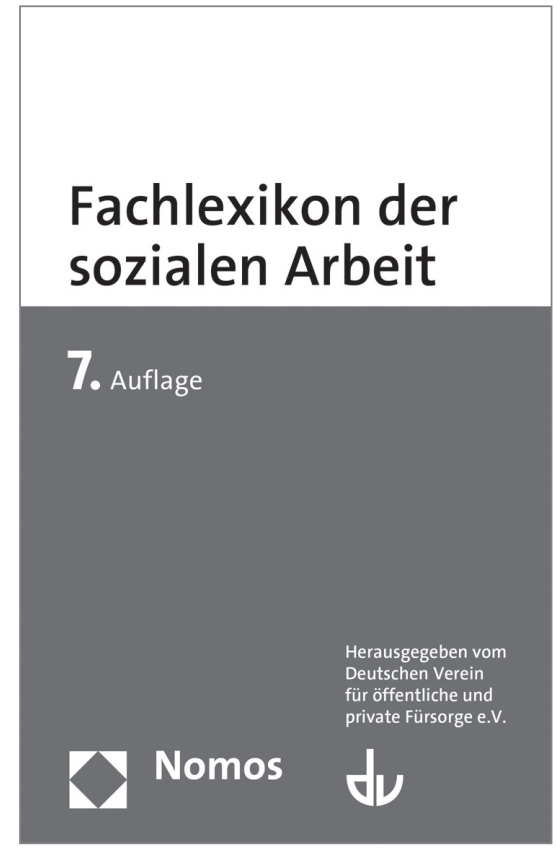

Quelle: Deutscher Verein für öffentliche und private Fürsorge e. V. (Hg.): Fachlexikon der sozialen Arbeit. 7. Auflage. Nomos Verlagsgesellschaft, Baden-Baden 2011. Ca. 1.200 Seiten. 44,- Euro. ISBN 9783-8329-5153-5. konkrete Wege erarbeiten, wie zum Beispiel der reale (oder empfundene) Stress gesenkt und die Widerstandsfähigkeit erhöht werden kann.

Dass es wichtig ist, die Widerstandsfähigkeit von Mitarbeitenden und Führungskräften zu stärken, um deren Motivation und Leistungskraft zu bewahren, das haben inzwischen viele Unternehmen erkannt. Deshalb werden Trainer und Coachs zunehmend von ihnen mit Anfragen konfrontiert wie: Können Sie unsere Mitarbeitenden dabei unterstützen, eine »dickere Haut " oder höhere Stressresistenz zu entwickeln?

Das tun viele Unternehmen vorwiegend aus betriebswirtschaftlichen Motiven. Denn auch sie wissen: Wenn ein Leistungsträger wegen eines Burn-outs längerfristig ausfällt, verursacht das nicht nur erhebliche Kosten. In unserer Organisation entsteht dann auch ein Chaos - was wiederum zu noch mehr Stress bei den noch gesunden Mitarbeitern führt.

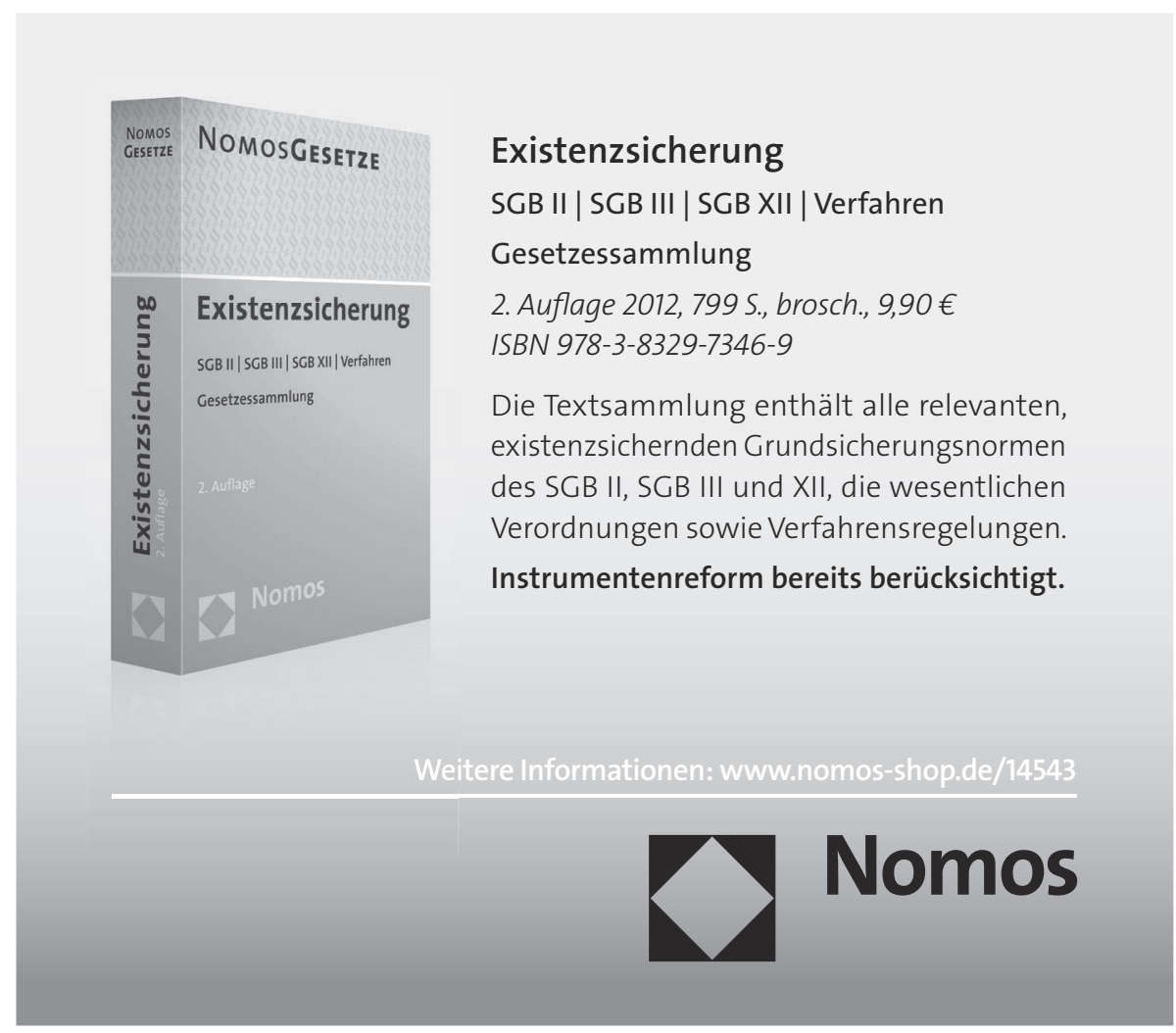

